\title{
Navigation by honey bees
}

2

Naïla Even ${ }^{1}$, Olivier Bertrand ${ }^{2}$, Mathieu Lihoreau
1 Institut National Polytechnique, Ecole Nationale Superieure d'Ingénieur en Art Chimique et Technologique (ENSIACET), Toulouse, France

2 Neurobiology and Center of Cognitive Interaction Technology (CITEC), Bielefeld University, Bielefeld, Germany

${ }^{3}$ Research Center on Animal Cognition (CRCA), Center for Integrative Biology (CBI); CNRS, University Paul Sabatier - Toulouse III, France

Corresponding author: mathieu.lihoreau@univ-tlse3.fr

\section{Definition}

Navigation by honey bees refers to the cognitive and behavioural mechanisms bees of the genus Apis use to move between a location and one or multiple goals. These involve the evaluation and integration of direction and distance cues as well as the learning of specific places. For honey bees, goals can be as diverse as the colony nest, a foraging site, a new potential nesting site, or an open-air mating area.

\section{Introduction}

The vast majority of the about 20,000 known bee species are central place foragers: adults collect and bring back resources (flower nectar and pollen, water, resins) in a stable nest where they raise their progeny (Michener, 2007). Foraging requires navigation skills to locate resources, travel between them, and return to the nest. Many bees must also navigate at some point in their life to find mating partners, a new suitable nesting site, or even to orient inside their dark nests.

About a century of research on insect navigation, starting with pioneering work of the Nobel Prize recipient Karl von Frisch (von Frisch, 1967), shows that these navigation behaviours are sustained by a cognitive "toolkit" (Wehner 2009) involving celestial cues (typically a sun compass), a distance estimator (odometer), a system for integrating both information (path integrator), and the ability to learn visual landmarks and specific places (see Figure $1 \mathrm{~A}$ and Glossary). For bees, these mechanisms sustain the expression of different types of spatial behaviours that change as individuals learn and gain experience with their environment.

In this chapter we describe these spatial behaviours in honey bees (Figure 2), a group of 11 species and 44 subspecies (Lo et al., 2010) exhibiting some of the most advanced levels of social forms in the animal kingdom characterised by a reproductive division of labour (fertile queens and drones, sterile workers), cooperative breeding and overlapping generations of adults. Navigation skills are required by individuals of the different castes (Seeley, 2012). The foragers collect food, water and resins from 
plants. The scouts must find new nesting sites. The reproductives must find mating partners for reproduction. And the in-hive workers move between important places in the nest, such as brood cells, honeycombs and the nest entrance. All these tasks require the ability to explore the environment, identify and learn places of interest and sometimes communicate spatial information to nestmates.

Here we focus in particular on the Western honey bee (Apis mellifera) as this species has a long history of domestication for honey production and crop pollination and is a model for the study of insect navigation. We discuss the different types of flights exhibited by foragers because they have been best described, especially since the development of automated tracking systems that record bee movements over several hundred meters (e.g. harmonic radar: Riley et al. 1996). However similar behaviours are expected by scout and reproductive bees. In-hive navigation has been less studied and is therefore not discussed.

\section{Exploration flights}

Western honey bees hatch in a dark nest, visually isolated from the outside environment. After about two weeks, they become foragers, which mean that they collect plant resources, sometimes scattered over several kilometers around the nest, to provision the colony. While foraging, the bee engages in different types of exploration flights during which it acquires visual memories of salient features of its environment, presumably in the form of snapshot memories (Collett et al., 2013), to guide future foraging trips.

On its first few excurions outside the colony nest, a bee engages in peculiar flight sequences known as 'learning flights' (Figure 1B), during which the bee learns the skyline panorama and visual landmarks associated with its nest location (Zeil, 2012). Learning flights are composed of many convoluted manoeuvers, loops, arcs, and zigzags during which the bee faces the nest. During the initial learning flight, the bee slowly increases the radii of the loops and arcs, distancing itself from the colony nest until a point when it returns home without bringing back any resources. On the next few flights, the bee flies a bit further, slowly gaining experience with the outside environment.

Once a visual memory of the nest location is acquired, the bee engages in 'orientation flights' (Figure 1C), this time turning back to the nest. The bee flies along extended loops anchored at the nest location and covering a narrow angular sector in a given direction. Successive orientation flights are longer loops covering other sectors of the environment until every direction has been covered (Capaldi et al., 2000). During 81 this process, the bee orients itself and searches for flower sites. If the bee is caught and released at an unfamiliar location outside the area covered by its previous flights, it engages in an extended search for its nest. By contrast, if the bee is released at a

84 familiar location, it flies straight back home (Degen et al., 2016). Drones (males) have also been shown to be faster to relocate the colony nest after several exploration 86 flights, allowing them to come back home from the drone congregation area if they 87 failed to mate a queen. 
Exploration flights are not exclusive to first trips outside the nest, but also occur during the foraging process. For instance, once a food resource is found, a forager may exhibit learning flights to learn the visual scene surrounding this new site so that it can more easily return to this site on subsequent foraging trips (Robert et al. 2018). When a resource is depleted, the bee searches for an alternative site through longer flights centered at the familiar depleted location. These exploration flights, composed of straight segments and turns, have Lévy characteristics, meaning that there is a larger proportion of long flight segments than one would expect from a normal distribution (i.e. a Brownian search) (Reynolds, 2008). This type of random search pattern is expected to be optimal to locate patchily distributed food sources, like plants, for animals with little knowledge of their environment.

101

\section{Homing flights}

103

At the end of every foraging trip, a forager must navigate back to its nest to unload its crop and feed the colony. 'Homing flights' are composed of two phases : a straight line flight to return to the nest area, and a convoluted flight to pinpoint of the nest entrance (Figure 1D).

To return home, the bee uses its estimation of directions and distances of the often turtuous inbound path toward a goal (e.g. feeding site) and integrates both information using a path integrator. In honey bees, directions are primarily estimated through the position of sun in the sky, constituting a sun compass. When the sun is not directly visible (e.g. during cloudy days), this information can be retrieved from the pattern of polarized light. Distances are estimated using an odometer through the quantity of visual information perceived on the retina of the compound eye while flying. It is possible to study this mechanism in bees by making them fly in narrow tunnels with black and white strips of different widths, thereby making them under- or overevaluate their real flight distances (Srinivasan et al., 2012).

When the crop (stomach) of the bee is full of nectar or its tarses are packed with pollen grains, its motivation to return home sets a new directional aim: the nest. A straight line return (also called 'beeline') is then indicated by the opposite of the integrated vector during the inbound journey. During the homing flight, the bee also integrates the distance and direction travelled, shrinking the integrated vector pointing to the nest. A central part of the bee brain, called the 'central complex', receives and integrates inputs of directions and distances from diverse internal senses like eyes, body hair, wing muscles, and other brain parts, and allows all these neural computations (Stone et al. 2017). The integration process is subject to noise accumulation during the journey of the bee. To reduce this error, foragers also refer to visual landmarks learned during previous flights to pinpoint directions and goals (Srinivasan 2011). Anything salient in the environment can be used as visual landmarks, even elongated structures on the ground such as rivers, edges and paths (Menzel et al. 2019).

130 The last bit of the homing flight (also known as 'view-based homing') is guided 131 by visual landscape cues. Since the seminal experiment of Niko Tinbergen (another 
132 Nobel Prize recipient) showing that digger wasps locate their nest entrance with 133 surrounding visual cues (Tinbergen 1932), bees have been challenged to pinpoint their 134 home in a plethora of scenarios. Objects may be displaced closer to or further away 135 from their nest, displaced to a new location, replaced with smaller or differently 136 coloured one, or even camouflaged (Dittmar et al., 2010). Based on these studies, we 137 know that bees learn relations between a myriad of visual cues and their nest entrance.

138 These relations are not necessarily explicit. Indeed, the use of panoramic skyline 139 snapshots around the nest entrance is sufficient to guide a simulated bee toward its 140 home (Towne et al., 2017).

Two navigational routines, path integration and visual guidance, jointly lead a bee home (Hoinville et al., 2018), but the importance of the routines depends on the context, such as the foraging experience of an individual bee. For instance, path integration tends to be preferred over visual landmarks in a novel environment. However when both navigational routines are conflicting, visual landmarks are favoured (Kheradmand and Nieh, 2019).

\section{Route following}

After several foraging trips, foragers tend to develop routes to efficiently return to known profitable feeding sites. Bees can famously be trained to learn straight line

152 outbound paths between the nest and a feeder providing large amounts of sucrose

153 solution (von Frisch, 1967). However, in many natural conditions, bees may visit 154 hundreds of flowers, sometimes dispersed over several kilometers, to fill their crop with nectar (honey bees have been observed homing after being released $11 \mathrm{~km}$ from their nest (Pahl et al., 2011)). When this is the case, foragers therefore need to develop 157 more complex circuits between multiple goals, a routing challenge analogous to the well-known Traveling Salesman Problem (TSP) in mathematics.

A naive honey bee forager first visits flowers in an unorderly sequence as it discovers them. However with experience, the bee can develop a stable route linking multiple flowers, a behaviour described in many other pollinators (e.g. bumblebees,

162 bats, hummingbirds) and known as trapline foraging (an analogy of the routes used

163 by trappers to check their traps; Figure 1D). This behaviour was discovered by 164 observing individual bees foraging in small arrays of artificial flowers within 100m from 165 the hive (Buatois and Lihoreau, 2016). As long as the artificial flowers were regularly 166 replenished with a sucrose reward, the bees learned to revisit them and adjusted their 167 visitation sequence in order to minimise overall travel distances, ultimately selecting the shortest possible route (i.e. thus solving the TSP).

Computational models attempting to decipher the cognitive mechanisms underpinning this navigational feat show that trapline development and optimisation can emerge based on the ability of bees to learn sequences of places using visual memories (Collett et al., 1993) and estimate travel distances with path integration

174 may sum the straight line distances between successively visited flowers and derive

175 the net length of the entire route. By comparing successive routes, the bee could thus 
176 increase its probability of re-using the vectors composing the shortest experienced

177 route (Lihoreau et al., 2012). Through trial and error, this simple learning process may

178 enable the development of good (if not optimal) routes in environments with different

179 numbers and spatial configurations of feeding sites (Reynolds et al., 2013).

\section{Place communication}

Once back in the hive, the honey bee forager can communicate the direction, distance and quality of a discovered feeding site via a sequence of movements known as the 'waggle dance' (von Frisch, 1967; Figure 3). Scout bees also use this communication system to indicate the location of a suitable nesting site (e.g. cavity, hive) to reach a consensus about the best available site before swarming (Seeley, 2012).

While this ability to recruit nestmates for profitable places was discovered by naturalists long ago (e.g. Aristotle), the symbolic meaning of the dance was finally decoded by Karl von Frisch. In 1919, von Frisch trained bees to forage on feeders containing sucrose solution placed around the hive. He observed that the foragers returning to the hive performed energetic rounds and were occasionally followed by other bees who would then fly to the communicated feeder. von Frisch described two types of behaviours referring them as "dances" depending on the distance of the food source to the hive. For feeders within 90 meters the hive, foragers performed a round movement, where for longer distances they performed a waggled movement following a eight-shaped figure. It is now acccepted that both dances are in fact a single behaviour, whose precision varies with the distance of the food source to the hive (Griffin et al. 2012).

218 Several Apis species from tropical Asia, such as the cavity nesting honey bee (e.g.

During the waggle dance, the forager waggles its abdomen and moves towards the direction of the food relative to the sun. In cavity nesting honey bees, like the Western honey bee, the invisible sun in the dark hive is encoded by the vertical vector facing up (i.e. against gravity). Therefore, the angle between the waggle trajectory and the vertical of the comb represents the angle of the food source and the sun. In open nesting honey bees, however, the dance occurs horizontally on the top of the single frame and the dancing bee faces directly the direction of the food or the future nest. In both open nesting and cavity nesting honey bees, the distance of the food source is communicated via the duration of the waggle movement that correlates with a distance in meters. This correlation and the precision of the duration slightly varies between species and subspecies of honey bees (Beekman et al. 2015). The rewarding value of the resource is communicated by the total duration that the bee performs repeating the eight-shape dance. Dancing for a longer time increases the probability to recruit foragers susceptible to be interested by the navigation instructions for a new resource (Seeley 2012).

\section{Migration}

Apis cerana), the giant honey bee (Apis dorsata) or the dwarf honey bee (Apis florea), 
do not remain in a stable nest but can abandon the nest or migrate over long distances to respond to local food shortage (Oldroyd 2009, Figure 2). Giant honey bees, for instance, have been reported to migrate to destinations up to $200 \mathrm{~km}$ away, stopping over in bivouac congregations to rest and forage. These bees can even sometimes return to the exact same tree after the migration period. During migration, Asian honey bees use dance communication to orient the swarm departure in the correct direction towards a new nest. In the case of long distance migrations, especially in open nesting giant honey bees, the direction is communicated but the distance is only indicated by a very long waggle signaling (Dyer and Seeley 1994). Sensory mechanisms used to migrate to a new location are similar to those used by honey bee foragers, including the sun compass, the optic flow, and the learning of visual landscape cues.

\section{Concluding remarks}

Honey bees share with other insects numerous sensory abilities and navigational strategies (see Glossary). Thus, they are key model species to study insect navigation. Understanding how a tiny brain, with about one million neurons, uses computations to navigate efficiently in three dimensions over large spatial scales, finds direct applications in algorithmic and robotics and may help understand how these mechanisms evolved in the more complex brains of vertebrates (Chittka and Niven, 2009). Some features described in mammal navigation studies, like the hypothesis of the representation of the global surrounding in a single mental map (i.e. cognitive map), influence debates about mental representations of space in insects (Menzel 2019). Even though no consensus has been yet reached, most studies show that path integration and visual guidance are sufficient to explain the complex spatial behaviour exhibited by honey bees and other insects (Webb 2019).

The study of the neural mechanisms underlying navigation in the insect brain may adress this important knowledge gap and clarify whether honey bees use cognitive maps. Deep brain structures start to be linked to navigational functions such as the central complex for path integration and the mushroom bodies for visual memories (Webb and Wystrach, 2016). Exploring brain function in vivo in a flying insect in its natural environment is still out of reach. However, recent advances in virtual reality setups (e.g. Buatois et al., 2018) and onboard neuron-stimulator or recorders (e.g. Sato et al., 2015) increase the possibilities to study navigation under tightly controlled, yet ecologically relevant, conditions while exploring brain function.

\section{References}

Beekman, M., Makinson, J. C., Couvillon, M. J., Preece, K., Schaerf, T. M. 2015. Honeybee linguistics - a comparative analysis of the waggle dance among species of Apis. Front Ecol Evol 3, 11.

Buatois, A., Lihoreau, M., 2016. Evidence of trapline foraging in honeybees. J Exp Biol 219, 2426-2429. 
Buatois, A., Flumian, C., Schultheiss, P., Avarguès-Weber, A., Giurfa, M. 2018. Transfer of visual learning between a virtual and a real environment in honey bees: the role of active vision. Front Behav Neurosci 12,139.

Capaldi, E.A., Smith, A.D., Osborne, J.L., Fahrbach, S.E., Farris, S.M., Reynolds, D.R., Edwards, A.S., Martin, A., Robinson, G.E., Poppy, G.M, Riley, J.R. 200. Ontogeny of orientation flight in the honeybee revealed by harmonic radar. Nature 403, 537-540.

Chittka, L., Niven, J. 2009. Are bigger brains better ? Curr Biol 19,R995-R1008.

Collett, M., Chittka, L., Collett, T.S. 2013. Spatial memory in insect navigation? Curr Biol. 23, R789, R800.

Degen, J., Kirbach, A., Reiter, L., Lehmann, K., Norton, P., Storms, M., Koblofsky M, Winter, S., Georgieva, P.B., Nguyen, H., Chamki, H., Meyer, H., Singh, P.K., Manz, G., Greggers, U., Menzel, R. 2016. Honeybees learn landscape features during exploratory orientation flights. Curr Biol. 26, 2800-2804.

Dittmar, L., Stürzl, W., Baird, E., Boeddeker, N., Egelhaaf, M. 2010. Goal seeking in honeybees: matching of optic flow snapshots? Journal Exp Biol 213, 29132923.

Dyer, F. C., Seeley, T. D. 1994. Colony migration in the tropical honey bee Apis dorsata f. (Hymenoptera: Apidae). Insectes Soc 41,129-140.

Collett, T.S., Fry, S.N., Wehner, R., 1993. Sequence learning by honeybees. J Comp Physiol A 172, 693-706.

Griffin, S. R., Smith, M. L., Seeley, T. D. (2012). Do honeybees use the directional information in round dances to find nearby food sources?. Anim Behav, 83, 1319-1324.

Kheradmand, B., Nieh, J.C. 2019. The role of landscapes and landmarks in bee navigation: A review. Insects 10, 342.

Lihoreau, M., Raine, N.E., Reynolds, A.M., Stelzer, R.J., Lim, K.S., Smith, A.D., Osborne, J.L., Chittka, L. 2012. Radar tracking and motion-sensitive cameras on flowers reveal the development of pollinator multi-destination routes over large spatial scales. PLoS Biol 10, e100139.

Lo, N., Gloag, R. S., Anderson, D. L., Oldroyd, B.P. 2010. A molecular phylogeny of the genus Apis suggests that the Giant Honey Bee of the Philippines, $A$. breviligula Maa, and the Plains Honey Bee of southern India, $A$. indica Fabricius, are valid species. System Entomol, 35, 226-233.

Menzel, R. 2019. The waggle dance as an intended flight: A cognitive perspective. Insects, 10, 424.

Menzel, R., Tison, L., Fischer-Nakai, J., Cheeseman, J.F., Sol Balbuena, M., Chen, X., landgraf, T., Petrasch, J., Polster, J., Greggers, U. 2019. Guidance of navigating honeybees by learned elongated ground structures. Front Behav Neurosci 12,322 .

Michener, C.D., 2007. The Bees of the World. The Johns Hopkins University Press, Balitmore, MA.

Oldroyd, B. P., Wongsiri, S. 2009. Asian Honey Bees: Biology, Conservation, and Human Interactions. Harvard University Press.

Pahl, M., Zhu, H., Tautz, J., Zhang, S. 2011. Large scale homing in honeybees. PloS One 6,e19669.

Reynolds, A.M., 2008. Optimal random Lévy-loop searching: New insights into the searching behaviours of central-place foragers. Europhys Lett article 20001. 
Reynolds, A.M., Lihoreau, M., Chittka, L., 2013. A simple iterative model accurately captures trapline formation by bumblebees across spatial scales and flower arrangements. PLoS Comp Biol 9, e1002938.

Riley, J.R., Smith, A.D., Reynolds, D.R., Ewards, A.S, Osborne, J.L., Williams, I.H., Carreck, N.L., Poppy, G.M. 1996. Tracking bees with harmonic radar. Nature 379, 29-30.

Robert, T., Frasnelli, E., Hempel de Ibarra, N., Collett, T.S. 2008. Variations on a theme : Bumblebee learning flights from the nest and from flowers. J Exp Biol 221: jeb172601

Sato, H., Vo Doan, T.T., Kolev, S., Huynh, N.A., Zhang, C., Massey. T.L., van Kleef, J., Ikeda, K., Abbeel, P., Maharbiz, M.M. 2015. Deciphering the role of a coleopteran steering muscle via free flight stimulation. Curr Biol 25, 798-803.

Seeley, T.D., 2010. Honeybee Democracy. Princeton University Press, Princeton, NJ. Srinivasan, M.V. 2011. Honeybees as a model for the study of visually guided flights, navigation, and biologically inspired robotics. Physiol Rev 91, 413-460.

Stone, T., Webb, B., Adden, A., Ben Weddig, N., Honkanen, A., Templin, R., Wcislo, W.T., Scimeca, L., Warrant, E., Heinze, S. An anatomically constrained model for path integration in the bee brain. Curr Biol 27, 3069-3085.

Tinbergen, N. 1932.Über die orientierung des bienenwolfes (Philanthus triangulum Fabr.). Z vergl Physiol 16, 305-334.

Towne, W. F., Ritrovato, A. E., Esposto, A., Brown, D. F. 2017. Honeybees use the skyline in orientation. J Exp Biol 220, 2476-2485.

von Frisch, K., 1967. The Dance Language and Orientation of Bees. Harvard University Press, Cambridge, MA.

Webb, B. 2019. The internal maps of insects. J Exp Biol 222: jeb188094

Webb, B., Wystrach, A. 2016. Neural mechanisms of insect navigation. Curr Opin Insect Sci 15,27-39.

Wehner, R., 2009. The architecture of the desert ant's navigational toolkit. Myrmec News. 12, 85-96.

Zeil, J. 2012. Visual homing: An insect perspective. Curr Opin Neurobiol 22, 285-293. 
343 Glossary: The honey bee navigation 'toolkit'. Concepts defined below are

344 highlighted in bold in the main text.

346 Central place foraging: Foraging behaviour consisting in collecting resources in the 347 environment and carrying them back home. In the case of honey bees, foragers make 348 back and forth trips between resource sites (food, water, resin) and their nest to feed 349 the colony.

Odometer: Mechanism enabling animals to estimate travel distances. In honey bees, distance estimation is mediated by the apparent motion of surrounding objects on the retina of the compound eye, also called 'optic flow'.

Panorama: Piece of visual information corresponding to a global visual cue, such as a landscape or a skyline. A panorama can also correspond to a combination of several visual landmarks (e.g. trees, buildings, rivers). Honey bees use panoramas for learning places such the location of their colony nest or a profitable food site.

Path integration: Method used by an animal for knowing its current position from a reference point, also known as 'dead reckoning'. Honey bees integrate odometry cues (distances) and sun compass (directions) to compute the vector pointing to a reference point (e.g. the nest) from their current position. This computation allows a honey bee to come back to the nest in a straight line.

Polarized light patterns: Rayleigh scattering creates patterns of polarized sunlight which are distributed in the sphere of the sky following concentric circles with the sun as a center (see Figure 1A). With photorecepteurs located in the dorsal area of their compound eyes, honey bees can detect direct sunlight as being the unpolarised area of the sky and can sense that polarisation increases when the e-Vectors (electric vectors of light) are further away from the sun along the concentric circles.

Snapshot: Learning of a 2D image at one point in time. For a honey bee, the memorised view corresponds to a specific orientation of the bee relative to the image,

Sun compass: Utilisation of the sun's position in the sky as a directional guide. For honey bees, the position of the sun can be perceived directly (when the sun is visible) or indirectly through the pattern polarized light (when the sun is not visible). To orient when the sun is visible, bees can use the light intensity (higher closer to the sun) and the chromatic gradients (toward green in solar region to UV in the antisolar region).

Trapline: Repeatable sequence of flower visits, staring and ending at the nest. With experience, honey bee foragers tend to develop traplines minimising overall travel distances between familiar feeding sites. These routes are based on individual experience. 
388 Visual landmark: Salient feature in the visual scene. Honey bees can use many types 389 of landmarks sapnning from 3D trees or buildings to 2D elongated patterns on the 390 ground (e.g. paths, edges, rivers, roads).

391

392

393 


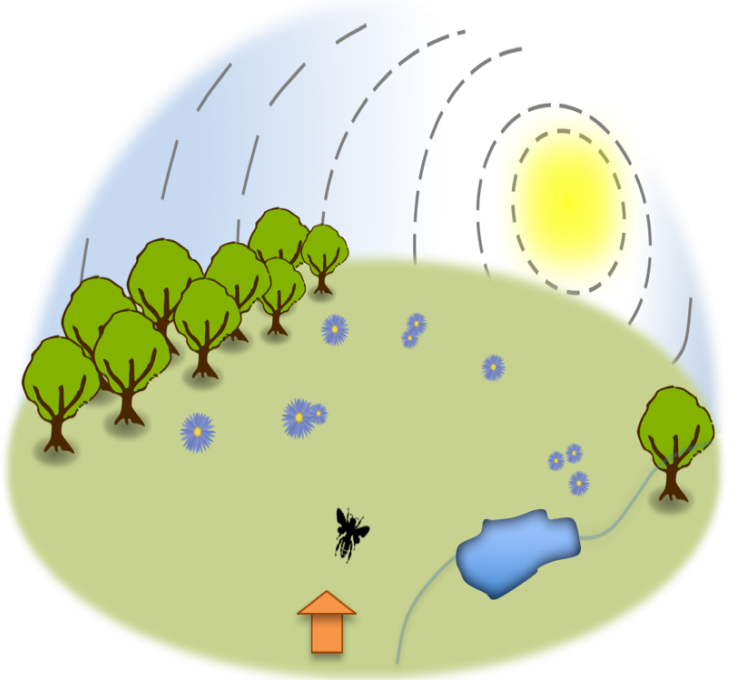

A. Environmental cues

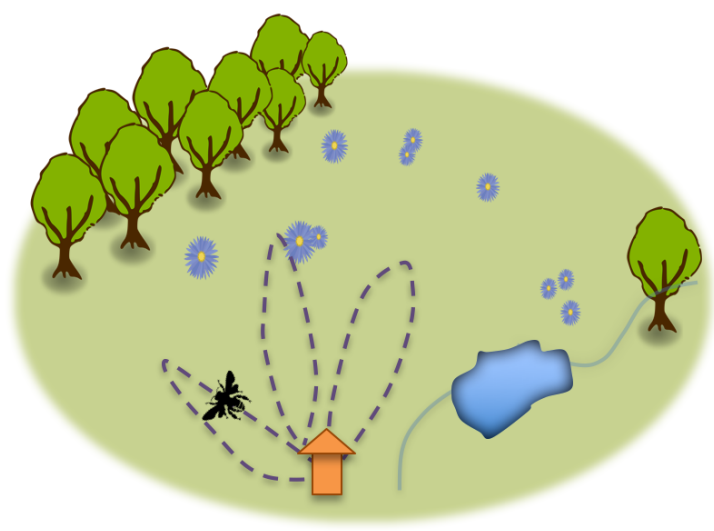

C. Orientation flights

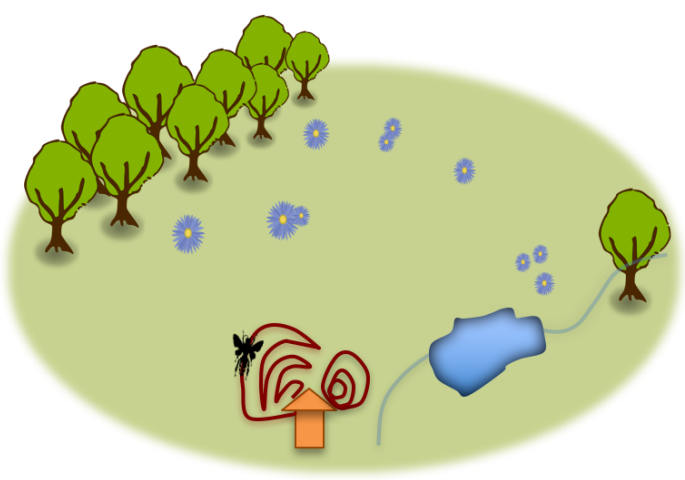

B. Learning flights

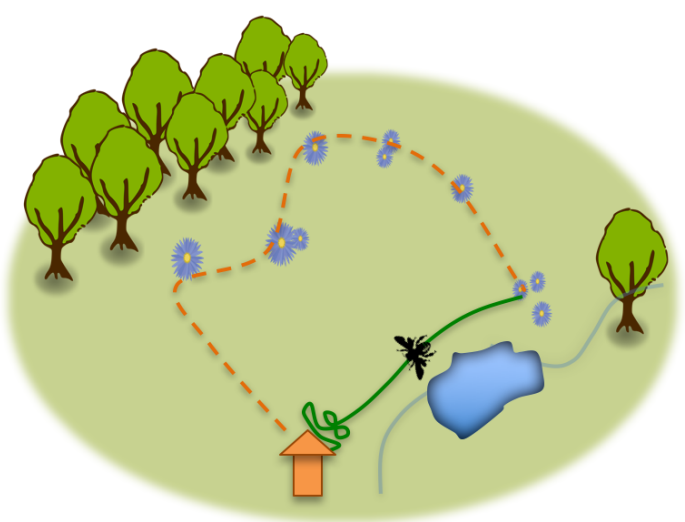

D. Route following and homing

Figure 1. A. Environmental cues used by honey bees to navigate. Bees use the position of the sun in the sky (or polarized light dashed lines under cloudy conditions, dashed lines) as compass. They learn visual landmarks (e.g. trees, rivers, paths) and views of broader visual cues like panoramas and skyline (i.e. snaphots). When closer to the goal, bees use local available information to pinpoint the goal (e.g. shape, colour, odours). B-D. Hypothetical examples of flight sequences performed by a forager as it gains experience. B. Learning flights: during its first excursions outside the hive the bee learns the visual scene caracterising the nest entrance using concentric circles increasing in size (red plain lines). C. Orientation flights : after a few trips, the bee starts exhibiting sequential exploration of sectors around the nest (orange dashed line) to find flower patches. D. Route following and homing: once experienced, the bee follows a multi-destination route (trapline) learnt from previous foraging trips (dashed orange line). The bee returns home following a home vector computed throug path integration 
411 (inbound in green). At the end of this homing flight the bee corrects eventual path 412 integration errors through small search loops to precisely locate the nest entrance.

413

414 


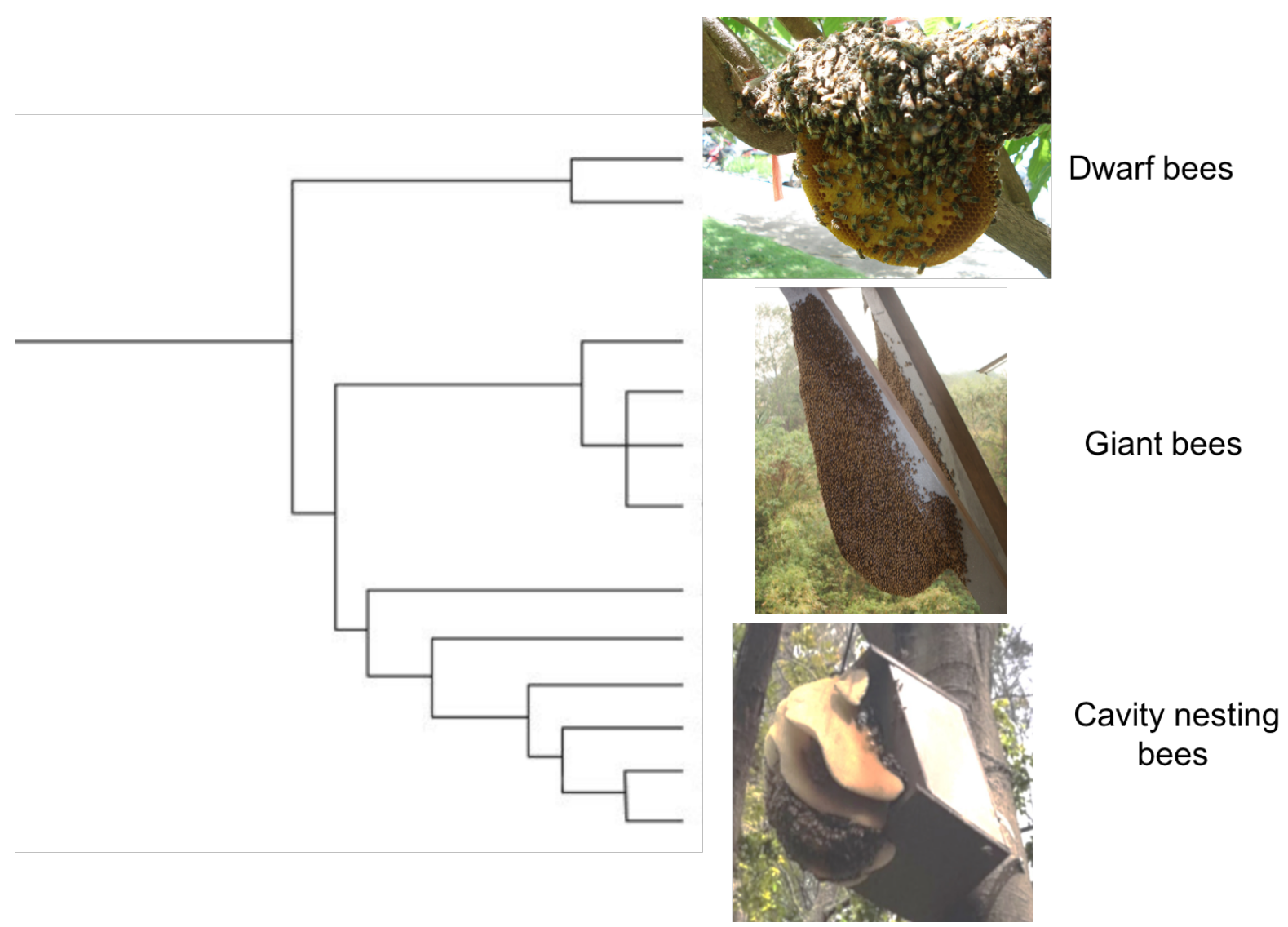

Figure 2. Honey bees contain 11 species. Dwarf open nesting honey bees like Apis florea are the most basal group and live mostly in Asia and Middle East. The open nesting giant honey bees like Apis dorsata are found only in Asia. The most recently derived group are the cavity nesting honey bees represented by six species: five of which are distributed in Asia. Apis mellifera has a natural distribution in Africa, Europe and the Middle East, but has been domesticated and distributed throughout the world. It is now found in the wild on all continents except Antarctica. Photo credits: Dwarf and cavity bees from Naïla Even, giant bees from Benjamin Oldroyd. 

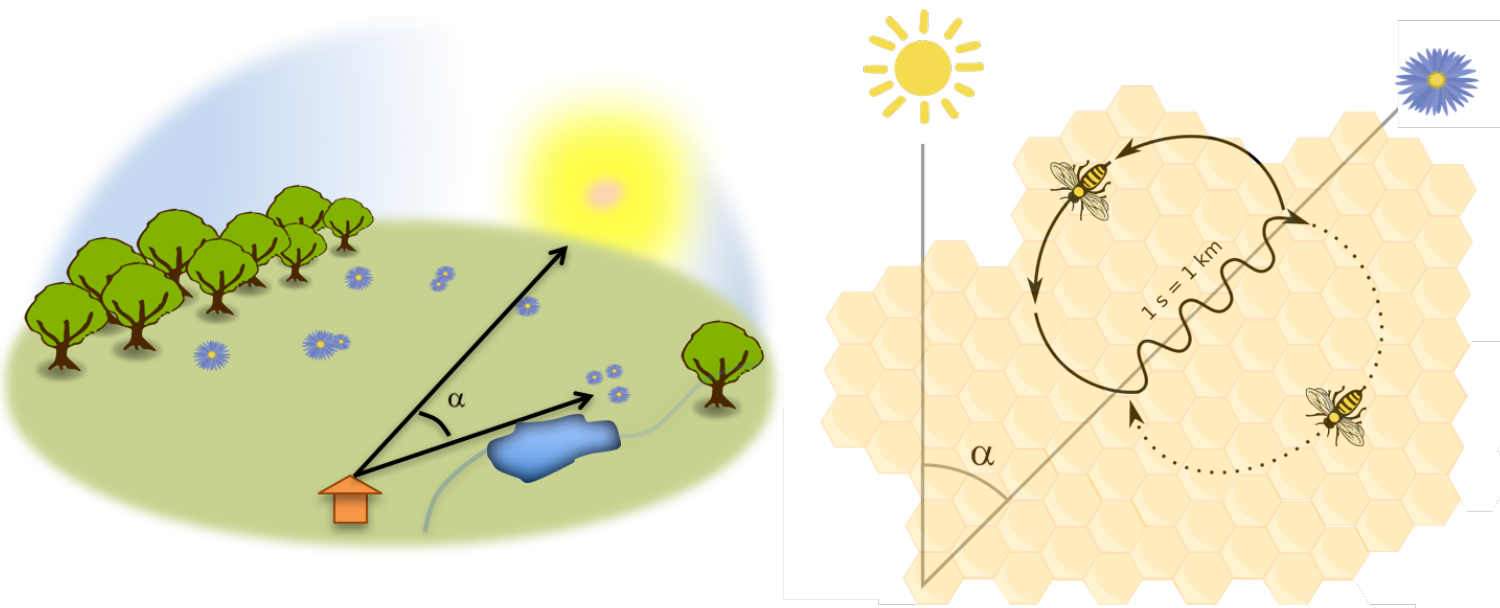

Figure 3. The waggle dance. Upon returning to the colony nest following a successful foraging trip, a honey bee forager can communicate the location of the discovered food site to its nestmates by performing a waggle dance. The bee communicates the direction of the resource by waggling in the direction of the resource relative to its angle with the sun ( ). The duration of the waggling movement is proportional to the distance of the food. The bee repeats the waggling movement coming back from the left and then from the right drawing the figure height. The more the dance is repeated the higher the quality of the resource. Any bee following the dance can thus integrate the information and leave the hive using the instruction from the dance. The waggle dance is also performed by scout honey bees to communicate the location of new nest site before swarming. Modified from Emmanuel Boutet (CC BY-SA 2.5).

Crosslink references (highlighted in yellow in the main text)

- Foraging by Honeybees

- View-Based Homing

- Central Place Foraging

- Insect Navigation

- Travel Salesman

- Cognitive Map

- Dead reckoning 